\title{
Zum Umgang mit Antisemitismus und anderen Formen Gruppenbezogener Menschenfeindlichkeit in der Berufsschule
}

\author{
Georg Lauss und Stefan Schmid-Heher
}

\section{Einleitung}

Am 20. Juni 2017 veröffentlichte eines der zentralen Publikationsmedien der USamerikanischen Alt-Right Bewegung Breitbart News einen Bericht mit dem Titel „Antisemitic views backed by half of Austria’s muslim youth“. Im Untertitel heißt es: „Almost 50 per cent of newly-arrived young Austrian Muslim migrants hold anti-Semitic views“". ${ }^{1}$ Illustriert wird der Bericht mit einem Foto, auf dem junge Palästinenser auf einer HamasDemonstration aus dem Jahr 2004 dabei zu sehen sind, wie sie einen Davidstern in Brand stecken.

Drei Tage zuvor war in der österreichischen Tageszeitung Der Standard ein Artikel mit dem Titel „Neue ,Gerüchte“ über Juden“ erschienen. ${ }^{2}$ Dieser Artikel wiederum bezieht sich (unter anderem) auf eine Studie über Haltungen zu Demokratie und Autoritarismus unter Wiener BerufsschülerInnen. ${ }^{3}$ Der Artikel präsentiert Antisemitismus als gesamtgesellschaftliches Problem. Zu lesen ist, dass $24 \%$ der SchülerInnen der Aussage zustimmen, dass Juden in Österreich zu viel Einfluss haben. Darüber hinaus wird die auffällig geringe Antwortbereitschaft bei dieser Frage thematisiert. Diskutiert werden auch gruppenspezifische Differenzen im Antwortverhalten. So weisen weibliche Befragte das antisemitische Klischee häufiger zurück als ihre männlichen Kollegen. Außerdem wird in der Tat darauf hingewiesen, dass $55 \%$ der muslimischen Jugendlichen, die sich positionieren, einen zu großen Einfluss von Juden in Österreich zu erkennen meinen. Sowohl in der Studie wie auch im Standard-Artikel wird vor Alarmismus gewarnt. Die Notwendigkeit von weiteren Untersuchungen wird hervorgehoben. Angesprochen wird auch, dass Prävention und Aufklärung über Antisemitismus in Schulen und darüber hinaus weiter einen hohen Stellenwert einnehmen müssen und keine Themen der Vergangenheit sind.

\footnotetext{
${ }^{1}$ Simon Kent, Antisemitic views backed by half of Austria's muslim youth, in: Breitbart News, [http://www.breitbart.com/jerusalem/2017/06/20/report-anti-semitic-views-backed-half-austrias-muslim-youth], eingesehen 23.10.2017.

2 Andras Szigetvari, Neue „Gerüchte“ über Juden, in: Der Standard, 17/18.6.2017, S. 4-5.

${ }^{3}$ Georg Lauss/Stefan Schmid-Heher, Politische Bildung an Wiener Berufsschulen. Demokratische und autoritäre Potentiale von Lehrlingen, in: Philipp Mittnik (Hrsg.), Empirische Einsichten in der politischen Bildung, Innsbruck 2017, S. 95-124.
} 
Nachdem die Verfasser des vorliegenden Beitrags auch die Autoren der von Breitbart zitierten Studie über Demokratie, Autoritarismus und Politische Bildung an Wiener Berufsschulen waren, werden im Folgenden einige für Theorie und Praxis der Politischen Bildung in der Migrationsgesellschaft dringliche Probleme behandelt. Im Zentrum steht dabei die Frage, wie im Rahmen von Politischer Bildung entschieden gegen Antisemitismus vorgegangen werden kann, ohne zugleich rassistischen und islamfeindlichen Diskursen Vorschub zu leisten. Welche Möglichkeiten gibt es, gegen bestimmte Formen Gruppenbezogener Menschenfeindlichkeit vorzugehen, die in manchen Bevölkerungsgruppen statistisch gesehen häufiger anzutreffen sind, ohne wiederum diese Gruppen insgesamt auf stereotype Weise zu stigmatisieren? Anders gefragt: Wie können sozialwissenschaftliche Studien dabei helfen, Probleme genauer zu beschreiben und zu lokalisieren, ohne dabei in die Falle einer „Versämtlichung “5 zu tappen, indem statistische Trends zu undifferenzierten und negativierenden Gruppenzuschreibungen werden?

Bei der Beantwortung dieser Frage wird folgendermaßen vorgegangen. Der zweite Abschnitt befasst sich mit didaktischen Zugängen zum Lerngegenstand Antisemitismus. Ohne die Notwendigkeit einer historischen Auseinandersetzung in Frage zu stellen, werden damit verbundene Probleme bei der Anknüpfung an die Lebenswelten von Jugendlichen aufgezeigt. Im Weiteren sollen Antisemitismus und Islamfeindlichkeit zueinander in Beziehung gesetzt werden, ohne sie dabei gleichzusetzen bzw. zu parallelisieren. Abschnitt drei beschreibt den methodischen Zugang von zwei quantitativen Erhebungen, deren Daten die empirische Grundlage der hier vorliegenden Überlegungen bilden. Im vierten Abschnitt werden die wichtigsten Ergebnisse der beiden Erhebungen zusammengefasst. In Abschnitt fünf vergleichen wir das Antwortverhalten von zwei Gruppen von BerufsschülerInnen. Die erste Gruppe setzt sich aus Befragten ohne Migrationshintergrund, in deren Haushalt ausschließlich Deutsch gesprochen wird, zusammen. Die zweite Gruppe umfasst Befragte, deren Haushaltssprache auf einen familiären Migrationshintergrund aus Ländern schließen lässt, in denen dem Islam eine gesellschaftlich und historisch prägende Rolle zukommt. Zuletzt wird geprüft, ob Antisemitismus in der Gruppe mit einem so verstandenen ,muslimisch geprägtem Familienhintergrund" weiter verbreitet ist, als in Gruppen, in deren Familien Serbisch oder Kroatisch gesprochen wird. Aufbauend auf den empirischen Ergebnissen werden in Abschnitt sechs didaktische Prinzipien für den Umgang mit Antisemitismus im Kontext von Berufsschulen dargelegt.

4 Ähnliche Artikel wie der oben zitierte in Breitbart News fanden sich auch in der Online Ausgabe der Jerusalem Post, wo dem Artikel ein Foto des Wachturms des ehemaligen Konzentrationslagers Mauthausen beigestellt war sowie im Daily Caller. - Benjamin Weinthal, Half of muslim youth in Austria hold antisemitic views, in: Jerusalem Post, 20.6.2017, [http://www.jpost.com/printarticle.aspx?id=497354], eingesehen 23.10.2017; Jake Rennie, Half of muslim minors in Austria hold Anti-Semitic views, in: The Daily Caller, 21.6.2017, [http://dailycaller.com/2017/06/21/half-of-muslimminors-in-austria-hold-anti-semitic-views-according-to-new-poll/], eingesehen 23.10.2017.

${ }_{5}^{5}$ Der Begriff wird Hedwig Dohm zugeschrieben. Vgl. Hedwig Dohm, Der Frauen Natur und Recht, Berlin 1876. 


\section{Didaktische Perspektiven auf Antisemitismus}

„Die Forderung, daß Auschwitz nicht noch einmal sei, ist die allererste an Erziehung. Sie geht so sehr jeglicher anderen voran, daß ich weder glaube, sie begründen zu müssen noch zu sollen." 6 Theodor W. Adorno formulierte seinen Anspruch an eine Erziehung nach Auschwitz als kategorischen Imperativ, als eine Maxime, die geltend macht, einzig aus menschlicher Vernunft zu resultieren. Daraus resultiert, dass die Infragestellung dieser Maxime bereits einen Tabubruch darstellt und sich somit moralisch delegitimiert. Keine der erdenklichen Rechtfertigungen, die mit der Verfolgung von Juden und Jüdinnen einhergingen und einhergehen, sollten damit Anspruch auf Gültigkeit erheben können. Sie alle haben gemeinsam, dass ,am Zusammenleben der Menschen einzig der Aspekt [interessiert], einen anderen dafür namhaft und schuldig zu machen, daß es nicht funktioniert“ und daher münden sie zwangsläufig , in einer Praxis der Verfolgung“. 7 So sehr die moralische Dimension von Antisemitismus in dieser Tatsache begründet ist, so herausfordernd ist eben diese moralische Dimension für einen pädagogischen Umgang mit dem Thema. Denn der Lerngegenstand Antisemitismus lässt sich nicht allein moralisch erschließen. Wenn historische Kontinuitäten und strukturelle Bedeutungen nicht wahrgenommen werden, ergibt sich auch aus einer moralischen Verurteilung keineswegs automatisch ein weiterer Reflexions- oder Handlungsbedarf. ${ }^{8}$ Barbara Schäuble konstatiert als Folge einer fehlenden Motivation zur Auseinandersetzung mit Antisemitismus eine „Spannung zwischen einem Sich-verpflichtet-Fühlen, VerpflichtetWerden und einer mangelnden Einsicht in Zusammenhänge "9, die durch das zwangsläufig damit verknüpfte Thema Holocaust zusätzlich aufgeladen wird. Auch von Borries stellt fest, dass der Holocaust angesichts seiner ,[[̈]]sthetisch-moralische[n] Widerlichkeit und kognitive[n] Unfassbarkeit“ aus geschichtsdidaktischer Sicht „zwar ein besonders wichtiger, aber kein lerngünstiger Gegenstand“ ist. ${ }^{10}$ Die Grundsätze des Beutelsbacher Konsens - Überwältigungsverbot, Kontroversitätsgebot und Interessenorientierung $^{11}$ - sind vor diesem Hintergrund nicht einfach einzulösen und dennoch muss sich jedes Bildungsangebot gegen Antisemitismus an ihnen messen lassen.

Schäuble macht vier zentrale Herangehensweisen antisemitismuskritischer Bildungsangebote aus: „Lernen über, Lernen aus, Lernen gegen und Lernen wegen

${ }^{6}$ Theodor W. Adorno, Erziehung zur Mündigkeit, Frankfurt a. M. 2013, S. 88.

7 Werner Konitzer, Einige Überlegungen. Antisemitismus und Moral, in: Mittelweg 3614 (2005), Heft 2, S. 24-35, hier S. 33 .

8 Barbara Schäuble, „Anders als wir“. Differenzkonstruktionen und Alltagsantisemitismus unter Jugendlichen, Berlin 2012, S. 412.

${ }^{9}$ Ebd., S. 411.

10 Bodo von Borries, Lernen und Lehren zum Holocaust - Konventionen, Irritationen, Provokationen?, in: epdDokumentation (2006), Heft 4/5, S. 48-65, hier S. 55.

${ }^{11}$ Hans-Georg Wehling, Konsens à la Beutelsbach? Nachlese zu einem Expertengespräch. Textdokumentation aus dem Jahr 1977, in: Benedikt Widmaier/Peter Zorn (Hrsg.), Brauchen wir den Beutelsbacher Konsens?. Eine Debatte der politischen Bildung, Bonn 2016, S. 19-27, hier S. 24. 
Antisemitismus". ${ }^{12}$ Während beim Lernen über Antisemitismus die Vermittlung von Wissen beispielsweise über Formen des Antisemitismus und die Verfolgungsgeschichte von Juden und Jüdinnen im Mittelpunkt steht, bezweckt der zweite Ansatz ein häufig von moralischen Erwartungen geprägtes „Lernen aus der Geschichte“. Die dritte Kategorie macht jene antisemitismusspezifischen Bildungsangebote aus, die sich zum Ziel setzen, gegen manifest oder latent antisemitische Einstellungen zu wirken bzw. eine entsprechende Prävention zu erzielen. Der vierte und letzte von Schäuble angeführte Bereich - Lernen wegen Antisemitismus - umfasst Bestrebungen im Zusammenhang mit verschiedensten Themen andere als antisemitische Deutungen und Orientierungsmuster anzubieten, „ohne dass dies notwendigerweise als ,Prävention’ ausgewiesen wird."13

Bildungsangebote der ersten beiden Kategorien bauen auf historischem Lernen. Dieses zielt darauf ab, „Kompetenz zur historischen Deutung“ zu gewinnen, sowie diese „historische Deutung in den Orientierungsrahmen der eigenen Lebenspraxis einzufügen und wirksam werden zu lassen“. ${ }^{14}$ Im Kontext des Unterrichtsfaches Politische Bildung an Berufsschulen stößt dieser grundsätzlich bedeutsame Zugang aber an seine Grenzen. Empirische Untersuchungen zeigen, dass die eigene Herkunft einen großen Einfluss auf die Wahrnehmung der NS-Geschichte hat und die Auseinandersetzung mit Geschichte in dieser Hinsicht der Migrationsgesellschaft Rechnung tragen muss. ${ }^{15}$ Für Jugendliche mit Migrationshintergrund ist der Nationalsozialismus in einem deutlich geringeren Ausmaß Teil ihrer eigenen Geschichte, als das für Jugendliche ohne Migrationshintergrund der Fall ist. ${ }^{16}$ Insbesondere in der Berufsschule erscheinen Bildungsangebote mit den Schwerpunktsetzungen Lernen über bzw. aus Antisemitismus aufgrund der mit ihnen verbunden Anforderungen an historisches Lernen weniger geeignet. Jugendliche, die nach der Erfüllung ihrer Schulpflicht eine Lehre im Dualen System beginnen, hatten nur von der sechsten bis zur achten Schulstufe Geschichtsunterricht. Im Rahmen des Pflichtgegenstandes Politische Bildung in der Berufsschule - für den über die zumeist dreijährige Lehrzeit hinweg insgesamt 80 Unterrichtsstunden vorgesehen sind - ist Geschichte nicht im Lehrstoff verankert, sondern lediglich als didaktisches Prinzip in den

12 Barbara Schäuble, „Über“, „aus“, „gegen“ oder „wegen“ Antisemitismus lernen? Begründungen, Themen und Formen politischer Bildungsarbeit in der Auseinandersetzung mit Antisemitismus, in: Richard Gebhart/Anne Klein/Marcus Meier (Hrsg.), Antisemitismus in der Einwanderungsgesellschaft. Beiträge zur kritischen Bildungsarbeit, Weinheim-Basel 2012, S. 174-191.

13 Ebd., S. 176

${ }^{14}$ Jörn Rüsen, Historisches Lernen. Grundlage und Paradigmen, Schwalbach/Ts. 2008, S. 64-65.

15 Viola Georgi, Entliehene Erinnerung. Geschichtsbilder junger Migranten in Deutschland, Hamburg 2003.

16 Während $29 \%$ der im Rahmen einer Studie zu Geschichtsbewusstsein und Identitätskonstruktionen von Jugendlichen mit Migrationshintergrund befragten Wiener SchülerInnen aus der AHS-Oberstufe bzw. aus berufsbildenden mittleren oder höheren Schulen mit zwei im Ausland geborenen Elternteilen kundtun, nichts mit dem Nationalsozialismus zu tun zu haben, weil sie sich nicht als ÖsterreicherIn fühlen, sind es unter jenen mit zumindest einem Elternteil aus Österreich nur $4 \%$. (Christiane Hintermann, Dissonante Geschichtsbilder? Empirische Untersuchung zu Geschichtsbewusstsein und Identitätskonstruktionen von Jugendlichen mit Migrationshintergrund in Wien, Wien 2007, S. 118.) 
Unterricht zu integrieren. ${ }^{17}$ Eine grundlegende historische Orientierung ist zwar für die Auseinandersetzung mit Antisemitismus ebenso wie für die Politische Bildung unverzichtbar, aber aufgrund der angeführten Rahmenbedingungen bleibt der Fokus im Weiteren auf Lernen ,gegen“ bzw. „,wegen“ Antisemitismus gerichtet.

Zur Erschließung von Antisemitismus als Lerngegenstand erscheinen die folgenden Fragen relevant: Wie kann dem „Brückenproblem“ - es bezeichnet die Schwierigkeit, eine „nachhaltige Begegnung zwischen Lernenden und Gegenstand anzubahnen, die an die Gesellschaftsbilder, Alltagstheorien und Erfahrungen von Lernenden anknüpft"18 _ entgegnet werden? Welche Rolle spielen Vorerfahrungen bzw. Voreinstellung der SchülerInnen?

Angesichts der oben beschriebenen Schwierigkeiten bei der Entwicklung von Lernmotivation verlangt eine didaktische Perspektive auf Antisemitismus eine lebensweltliche Orientierung im Sinne des didaktischen Prinzips der Adressatenorientierung. Dieses fokussiert auf ,interaktive ,Sinndeutung“ der ,miteinander handelnden Personen““. ${ }^{19}$ Warum man über bzw. aus Antisemitismus lernen soll, erschließt sich nicht allein aus moralischen Ansprüchen. Auch wenn Antisemitismus moralisch verurteilt wird, geht damit weder automatisch eine Lernmotivation noch ein Lernprozess einher. ${ }^{20}$ Die Beziehung zum Lerngegenstand Antisemitismus ist so zu gestalten, dass die SchülerInnen ihre Deutungen zum Ausdruck bringen können. Insofern anzunehmen ist, dass die Deutungen in der Regel auch antisemitisch bzw. von antisemitischen Stereotypen geprägt sind, muss zunächst vonseiten der PädagogInnen die moralische Dimension des Themas zugunsten einer rekonstruktiven Erkenntnishaltung hintangestellt werden. Sie sind gefordert, die „lebensweltgebundenen Topoi ihrer Adressatinnen und Adressaten [zu] analysieren“, um so „ein Wissen um subjektive Begründungen" $21 \mathrm{zu}$ erlangen und diese im Weiteren thematisieren $\mathrm{zu}$ können. Hinderlich wäre die Wahrnehmung solcher antisemitischer Deutungen als

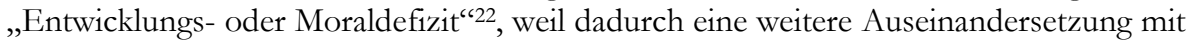
den Motiven und Interessenlagen der Jugendlichen blockiert wird. Genauso wenig, wie fachliche Fehlkonzepte aus der Lebenswelt „von wissenschaftlichen Konzeptionen einfach abgelöst werden“ 23 können, lässt sich ein antisemitisches Verständnis einfach durch ein anderes austauschen.

17 Unter „Besondere didaktische Grundsätze für den Pflichtgegenstand Politische Bildung“ ist angeführt, dass „,[z]eitgeschichtliche Entwicklungen [...] unter Beachtung der Bedeutung der historischen Dimension der zu behandelnden Themenbereiche, insbesondere der Demokratie und Menschenrechte, in den Unterricht zu integrieren [sind].“ (BGBl. II Nr. 211/2016)

18 Andreas Petrik, Der heimliche politikdidaktische Kanon, in: Ingo Juchler (Hrsg.), Unterrichtsleitbilder in der politischen Bildung, Schwalbach/Ts. 2012, S. 71-85, hier S. 72.

19 Andreas Petrik, Adressatenorientierung, in: Wolfgang Sander (Hrsg.), Handbuch politische Bildung, Bonn 2014, S.

241-248, hier S. 241.

20 Schäuble, Anders, S. 412

${ }^{21}$ Ebd., S. 428-429

22 Ebd., S. 419

${ }^{23}$ Dirk Lange, Kernkonzepte des Bürgerbewusstseins, in: Georg Weißeno (Hrsg.), Politikkompetenz. Was Unterricht 
Als Ergebnis mangelnder lebensweltlicher Anknüpfungspunkte in der schulischen Auseinandersetzung mit Antisemitismus bleibt in vielen Fällen, dass vor allem gelernt wird, sich entsprechend der Moralvorstellungen des jeweiligen Umfelds zu Antisemitismus zu äußern. ${ }^{24}$ In einer Migrationsgesellschaft gestaltet sich dieses Umfeld in einem zusätzlichen Maße divers und es lassen sich vielfältige Sinn- und Bedeutungszusammenhänge antisemitischer Einstellungen ausmachen. Solche könnten beispielsweise beabsichtigte Provokation, die Thematisierung des Nahostkonflikts, verkürzte Globalisierungskritik oder Verschwörungstheorien sein. Diesen Zusammenhängen kommt in Lernprozessen eine zentrale Bedeutung zu. Darüber hinaus ist ein gesellschaftlicher Diskurs, in dem die Thematisierung von antisemitischen Einstellungen bei MuslimInnen die Funktion erfüllt, die Mehrheitsgesellschaft und nichtmuslimische Minderheiten zu entlasten, in zweifacher Hinsicht problematisch: Er lagert das Problem aus, blockiert damit von vornherein Lernprozesse und dient der moralischen Empörung über „die Anderen“. Mit anderen Worten: Antisemitismus als Problem der Mehrheitsgesellschaft wird dadurch verdrängt und antimuslimische Ressentiments finden eine im öffentlichen Diskurs weithin akzeptierte Ausdrucksweise.

\section{Methode}

Die Umfragedaten, auf denen dieser Beitrag beruht, wurden im ersten Halbjahr 2016 erhoben. Befragt wurden einerseits Wiener BerufsschülerInnen ${ }^{25}$ und andererseits Studierende der Pädagogischen Hochschule Wien (PH Wien) ${ }^{26}$.

Zunächst wurden im Februar und März 2016 rund 700 Lehrlinge aus insgesamt 25 verschiedenen Lehrberufen an zehn Wiener Berufsschulen befragt. Bei der Zusammenstellung des Samples wurde darauf geachtet, dass aus Lehrberufen mit vielen Lehrlingen auch verhältnismäßig viele BerufsschülerInnen befragt werden und zugleich eine breite Streuung über verschiedenste Branchen erreicht werden kann. Aus rechtlichen Gründen waren alle TeilnehmerInnen mindestens 18 Jahre alt. Für die Auswertung wurden nur die Antworten jener 636 SchülerInnen herangezogen, die bis zur letzten Frage an der Umfrage teilnahmen. Eine Genehmigung des Stadtschulrats für Wien, sowie die Zusammenarbeit mit den Direktionen und Lehrenden der ausgewählten Schulen ermöglichte die Befragung während der Unterrichtszeit, wobei dafür nach Möglichkeit eine Stunde aus Politischer Bildung verwendet wurde. Vergleichsdaten zu den Antworten der BerufsschülerInnen wurden im März und April 2016 unter Studierenden der PH Wien erhoben. Insgesamt wurden 1006 Studierende, bei denen sichergestellt war, dass

zu leisten hat, Bonn 2008, S. 245-258, hier S. 246.

24 Schäuble, Über, S. 175

${ }^{25}$ Lauss/Schmid-Heher, Berufsschule.

26 Georg Lauss/Stefan Schmid-Heher, Politische Bildung und demokratierelevante Einstellungsmuster von angehenden Lehrenden. Eine quantitative Studie, in: R\&E Source (2017), Heft Tag der Forschung, S. 1-13. 
sie zum Zeitpunkt der Befragung schon eine Lehrveranstaltung zu Politischer Bildung besucht hatten, zur Teilnahme an der Studie aufgefordert. 334-mal wurde der Fragebogen aufgerufen und 302-mal bis zur letzten Frage beantwortet und abgeschickt. Die Daten wurden mittels Onlineumfrage via Limesurvey über Smartphones erhoben. Der Fragebogen war so konzipiert, dass das vollständige Ausfüllen zwischen zehn und 15 Minuten dauern sollte. Da die Fragen innerhalb der einzelnen Abschnitte jeweils per Zufallsgenerator gereiht wurden, sind Reihenfolgeeffekte auszuschließen. Die Datensätze wurden nach Beendigung der Erhebungsphase mittels SPSS statistisch ausgewertet.

Der Fragebogen besteht aus drei Abschnitten mit insgesamt 37 Items, wobei sich im Abschnitt A 17 Items mit politischen Einstellungen und sechs Items mit der politischen Aktivität befassen, im Abschnitt B acht Items mit der Wahrnehmung von Politischer Bildung und im Abschnitt $\mathrm{C}$ sechs Items mit demographischen Angaben zur Person. Die Abfrage politischer Einstellungsmerkmale baut in Grundzügen auf den Merkmalen der autoritären Persönlichkeit auf und gliedert sich in Konstrukte zu politischem Autoritarismus, Autoritarismus, Anomie und Gruppenbezogener Menschenfeindlichkeit. Einzelne Konstrukte sind in Dimensionen unterteilt, denen jeweils ein bis drei Items zugeordnet sind. Das Konstrukt Autoritarismus umfasst die Dimensionen Unterwerfung und Aggression; Anomie gliedert sich in Orientierungslosigkeit, politische Machtlosigkeit sowie Zukunftsperspektive und Gruppenbezogene Menschenfeindlichkeit wird in Anlehnung an Heitmeyer in den Dimensionen Zuwanderungsängste, Antisemitismus, Islamfeindlichkeit sowie Sexismus erfasst. ${ }^{27}$ Bei der Auswahl der Items wurde $\mathrm{zu}$ wesentlichen Teilen auf die Studie „NS-Geschichtsbewusstsein und autoritäre Einstellungen in Österreich“ 28 sowie auf die ländervergleichende Studie „Historical totalitarian experiences, authoritarian potential and democratic values in Austria, Poland, Hungary and the Czech Republic" ${ }^{\text {"29 }}$ zurückgegriffen. Ebenso wurde auf deutsche und österreichische Jugendwertestudien Bezug genommen. ${ }^{30}$

Die Fragebögen bestehen weitestgehend aus Likert-Skalen mit vier Antwortmöglichkeiten (stimme sehr zu ... stimme gar nicht zu) und der Möglichkeit ,keine Antwort“. Die überwiegende Mehrzahl der Konstrukte und Dimensionen besteht aus sowohl positiv als auch negativ formulierten Items. Dies ist vor allem im Bereich der Autoritarismusforschung ein entscheidender Punkt. Denn wenn man annehmen muss, dass autoritäre Charaktere dazu tendieren Fragen eher zustimmend zu beantworten,

${ }^{27}$ Wilhelm Heitmeyer, Gruppenbezogene Menschenfeindlichkeit (GMF) in einem entsicherten Jahrzehnt, in: Ders. (Hrsg.), Deutsche Zustände. Folge 10, Berlin 2015, S. 15-41

${ }^{28}$ Oliver Rathkolb/Martina Zandonelle/Günther Ogris, NS-Geschichtsbewußtsein und autoritäre Einstellungen in Österreich, Wien 2014, [http://www.sora.at/fileadmin/downloads/projekte/2014_Presseunterlage_Geschichtsbewusstsein-undautoritaere_Einstellungen.pdf], eingesehen am 2.11.2016

${ }^{29}$ Oliver Rathkolb/Günther Ogris, Historical totalitarian experiences, authoritarian potential and democratic values in Austria, Poland, Hungary and the Czech Republic, Wien 2008.

30 Bernhard Heinzlmeier/Phillip Ikrath, Jugendwertestudie 2011, Wien 2012, S. 55. 
würde man die Ergebnisse noch akzentuieren, wenn man StudienteilnehmerInnen überwiegend autoritäre Aussagen vorlegt.

\section{Demokratische und autoritaristische Potentiale von Wiener BerufsschülerInnen und Lehramtsstudierenden an der PH Wien}

Der folgende Abschnitt beginnt mit einer kurzen Einführung in das Konzept der autoritären Persönlichkeit. Danach werden zentrale Ergebnisse der oben beschriebenen Studien zusammengefasst und das Antwortverhalten von zumindest 18-jährigen BerufsschülerInnen und von Lehramtsstudierenden in Wien verglichen. In den Nachkriegsgesellschaften Europas und Nordamerikas, die den Umschlag demokratischer Systeme in Autokratie und Totalitarismus noch lebhaft vor Augen hatten, entstanden eine Reihe von Untersuchungen zur politischen Sozialisation von Kindern und jungen Erwachsenen. ${ }^{31}$ Diese erreichten in den 1970er-Jahren ihren Höhepunkt. ${ }^{32}$ Im deutschsprachigen Raum war es insbesondere die „Frankfurter Schule“ um Horkheimer und Adorno, die sich mit Fragen der politischen Sozialisation und demokratischer Erziehung in Gesellschaft, Schule und Familie auseinandersetzte. ${ }^{33}$ Grundlegend dabei war die erstmals im US-amerikanischen Exil entstandene Studie zum autoritären Charakter. ${ }^{34}$ Personen mit autoritären Einstellungsmustern bereitet es Schwierigkeiten, den grundlegenden Konflikt zwischen den Werten sozialer Anpassung und persönlicher Autonomie $\mathrm{zu}$ lösen. ${ }^{35}$ Das Vertrauen in demokratische Prinzipien ist schwach ausgeprägt. Dies wirkt sich in einer gesteigerten Bereitschaft zur Unterwerfung einerseits und aggressiv-dominantem Verhalten gegenüber Schwächeren andererseits aus. Kritiklose Befolgung der Anweisung von Autoritäten geht demnach einher mit einer idealisierenden Überbewertung der Eigengruppe und Abwertung des Fremden. Personen und Gruppen, die gesellschaftlich etablierten Normen widersprechen, werden zu Zielen von Aggression. Zukunftsangst und Orientierungslosigkeit steigern das Bedürfnis nach konformistischem Sozialverhalten. ${ }^{36}$

31 David Easton/Jack Dennis, Children in the Political System, New York 1969; Herbert Hyman, Political Socialization. A Study in the Psychology of Political Behavior, Glencoe 1959; Fred Greenstein, Children and Politics, New Haven 1965; Harold Lasswell, Democratic Character, in: Ders. (Hrsg.), Political Writings of Harold Lasswell, Glencoe 1951, S. 465-525.

32 Robert Dudley/Alan Gitelson, Civic Education, Civic Engagement, and Youth Civic Development, in: Political Science and Politics 36 (2003), Heft 2, S. 263-267; Richard Niemi/Mary Hepburn, The Rebirth of Political Socialization, in: Perspectives on Political Science 24 (1995), Heft 1, S. 7-16.

33 Theodor W. Adorno, Studien zum autoritären Charakter, Frankfurt a. M. 2013; Karlheinz Rebel (Hrsg.), ZwangAutorität-Freiheit in der Erziehung. Texte zum Autoritätsproblem, Berlin 1967.

34 Theodor W. Adorno u. a., The Authoritarian Personality, Oxford 1950.

35 Stanley Feldman, Die Konzeptualisierung und die Messung von Autoritarismus. Ein neuer Ansatz, in: Susanne Rippl/Christian Seipel/Angela Kindervater (Hrsg.), Autoritarismus, Wiesbaden 2000, S. 239-260; Stanley Feldman, Enforcing Social Conformity, in: Political Psychology 24 (2003) Heft 1, S. 41-74.

${ }^{36}$ Bob Altemeyer, The Authoritarian Specter, Cambridge (MA) 1996. 
Das Vertrauen in demokratische Prinzipien ist in der Gruppe der Lehrlinge insgesamt schwächer ausgeprägt als unter Lehramtsstudierenden. Lediglich $68 \%$ halten Demokratie für die beste Regierungsform. Unter Lehramtsstudierenden sind es immerhin 91 \%. Bei einer anderen Frage aus dem Konstrukt politischer Autoritarismus ergibt sich ein ähnliches Bild. Fast die Hälfte der Lehrlinge wünscht sich „an der Spitze eines Staates eine starke Persönlichkeit, die sich nicht um ein Parlament und Wahlen kümmern muss". Unter den Lehramtsstudierenden sind es mit $31 \%$ etwas weniger. Für die Vorstellung, dass ExpertInnen und nicht eine gewählte Regierung entscheiden sollen, was am besten für das Land ist, findet sich in beiden Gruppen eine deutliche Mehrheit. Demonstrationsverbote an neuralgischen Verkehrsadern, wie der Wiener Ringstraße, finden eine relative Mehrheit unter Wiener Lehrlingen. Unter PH-Studierenden stellen sich dreimal mehr Befragte gegen ein Verbot, als es befürworten.

\begin{tabular}{|c|c|c|c|c|c|}
\hline $\begin{array}{l}\text { Politischer Autoritarismus: } \\
\text { Lehrlinge (BS) und PH-Studierende (PH) im Vergleich }\end{array}$ & & ++ & + & - & -- \\
\hline \multirow{2}{*}{$\begin{array}{l}\text { Die Demokratie ist die beste Regierungsform, auch wenn sie } \\
\text { Probleme mit sich bringen mag. }\end{array}$} & BS & 27 & 41 & 18 & 6 \\
\hline & $\mathrm{PH}$ & 56 & 35 & 6 & 1 \\
\hline \multirow{2}{*}{$\begin{array}{l}\text { An der Spitze eines Staates sollte eine starke Persönlichkeit } \\
\text { stehen, die sich nicht um ein Parlament und Wahlen } \\
\text { kümmern muss. }\end{array}$} & BS & 20 & 27 & 24 & 16 \\
\hline & PH & 11 & 20 & 26 & 33 \\
\hline \multirow{2}{*}{$\begin{array}{l}\text { ExpertInnen und nicht die Regierung sollten entscheiden, } \\
\text { was das Beste für das Land ist. }\end{array}$} & BS & 19 & 34 & 26 & 10 \\
\hline & $\mathrm{PH}$ & 16 & 39 & 24 & 10 \\
\hline \multirow{2}{*}{$\begin{array}{l}\text { Ein Demonstrationsverbot auf der Ringstraße halte ich für } \\
\text { gerechtfertigt. }\end{array}$} & BS & 20 & 21 & 20 & 18 \\
\hline & $\mathrm{PH}$ & 7 & 14 & 24 & 40 \\
\hline
\end{tabular}

Wie zuvor beschrieben, sind Aggression, Unterwerfung und Konventionalismus Kennzeichen für autoritäre Einstellungsmuster. Auch in diesem Bereich unterscheiden sich die befragten Gruppen durchgängig. Zwei Drittel der BerufsschülerInnen stimmen der Aussage zu, dass Disziplin und Gehorsam in der Gesellschaft wieder wichtiger werden müssen. Unter PH Studierenden sind es vier von zehn. Allerdings befürworten auch $51 \%$ der Studierenden härteres Vorgehen gegen Unruhestifter, um Recht und Ordnung zu bewahren. Unter Lehrlingen sind es mehr als $70 \%$. $41 \%$ der Lehramtsstudierenden können sich vorstellen, manchmal auch Gnade vor Recht ergehen zu lassen. Unter Wiener BerufsschülerInnen ist das für $26 \%$ der Befragten eine Option. 


\begin{tabular}{|l|l|l|l|l|l|}
\hline $\begin{array}{l}\text { Autoritarismus: } \\
\text { Lehrlinge (BS) und PH-Studierende (PH) im Vergleich }\end{array}$ & & ++ & + & - & -- \\
\hline $\begin{array}{l}\text { Es braucht Leute, die Neues ausprobieren, auch wenn sie } \\
\text { dabei Regeln verletzen. }\end{array}$ & BS & 22 & 33 & 26 & 11 \\
\cline { 2 - 6 } & PH & 16 & 38 & 27 & 8 \\
\hline $\begin{array}{l}\text { Disziplin und Gehorsam müssen in unserer Gesellschaft } \\
\text { wichtiger werden. }\end{array}$ & BS & 41 & 24 & 15 & 5 \\
\cline { 2 - 6 } & PH & 12 & 28 & 33 & 16 \\
\hline \multirow{2}{*}{$\begin{array}{l}\text { Um Recht und Ordnung zu bewahren, sollte man härter } \\
\text { gegen UnruhestifterInnen vorgehen. }\end{array}$} & BS & 36 & 35 & 15 & 6 \\
\cline { 2 - 6 } & PH & 20 & 31 & 30 & 10 \\
\hline $\begin{array}{l}\text { Anstatt hart zu strafen, sollte man manchmal Gnade walten } \\
\text { lassen. }\end{array}$ & BS & 8 & 18 & 30 & 33 \\
\cline { 2 - 6 } & PH & 7 & 34 & 29 & 19 \\
\hline
\end{tabular}

Eine Tendenz zur Gruppenbezogenen Menschenfeindlichkeit wurde in den Kategorien allgemeine Zuwanderungsängste, Islamfeindlichkeit, Antisemitismus und Sexismus abgefragt. Während sich eine relative Mehrheit der BerufsschülerInnen durch Zuwanderung beruflich bedroht fühlt, trifft das nur für $12 \%$ der PH-Studierenden zu. Mehr als die Hälfte der Lehrlinge sieht in Zuwanderung einen Nachteil für die österreichische Gesamtwirtschaft. Unter Lehramtsstudierenden teilen nicht ganz ein Drittel der Befragten diese Einschätzung. Weniger als ein Fünftel der Studierenden gibt an, mit Menschen aus dem eigenen Herkunftsland weniger Probleme zu haben als mit anderen. Unter BerufsschülerInnen sind beide Gruppen in etwa gleich groß. Sollten MuslimInnen in Österreich das Recht auf eigene Kulturorganisationen haben, solange sie sich an die Gesetze halten? Unter angehenden LehrerInnen sprechen sich immerhin $25 \%$ gegen dieses Recht aus. Unter Lehrlingen sind es sogar $45 \%$. Dies ist insbesondere deshalb bemerkenswert, weil hier durch die Verfassung geschützte Grundrechte wie das Recht auf Vereins- und auf Versammlungsfreiheit (Art. 12 StGG) und das Recht auf Glaubens- und Gewissensfreiheit einschließlich der Freiheit der Religionsausübung (Art 14 und 16 StGG; Art. 9 EMRK) berührt werden. Auch antisemitische Einstellungen sind unter den befragten Studierenden deutlich seltener anzutreffen. $5 \%$ finden, dass Juden in Österreich zu viel Einfluss haben. Unter BerufsschülerInnen vertreten immerhin ein Viertel der Befragten diese Ansicht. Und auch bei der Frage nach beruflicher Gleichstellung von Mann und Frau lassen sich Unterschiede feststellen. Während unter BerufsschülerInnen $29 \%$ der Befragten sich dagegen aussprechen, dass Frauen häufiger Führungspositionen übernehmen sollten, sind es unter PH-Studierenden nur $13 \%$. 


\begin{tabular}{|c|c|c|c|c|c|}
\hline $\begin{array}{l}\text { Gruppenbezogene Menschenfeindlichkeit: } \\
\text { Lehrlinge (BS) und PH-Studierende (PH) im Vergleich }\end{array}$ & & ++ & + & - & -- \\
\hline \multirow{2}{*}{$\begin{array}{l}\text { Meine beruflichen Chancen sinken durch steigende } \\
\text { Zuwanderung. }\end{array}$} & BS & 23 & 24 & 25 & 19 \\
\hline & $\mathrm{PH}$ & 5 & 7 & 32 & 53 \\
\hline \multirow{2}{*}{$\begin{array}{l}\text { ZuwandererInnen sind im Allgemeinen gut für die } \\
\text { österreichische Wirtschaft. }\end{array}$} & BS & 9 & 25 & 28 & 27 \\
\hline & $\mathrm{PH}$ & 16 & 40 & 25 & 7 \\
\hline \multirow{2}{*}{$\begin{array}{l}\text { Mit Menschen aus meinem eigenen Herkunftsland habe ich } \\
\text { weniger Probleme als mit anderen. }\end{array}$} & BS & 25 & 21 & 21 & 24 \\
\hline & $\mathrm{PH}$ & 7 & 12 & 24 & 51 \\
\hline \multirow{2}{*}{ Juden haben in Österreich zu viel Einfluss. } & BS & 15 & 9 & 20 & 35 \\
\hline & $\mathrm{PH}$ & 1 & 4 & 17 & 58 \\
\hline \multirow{2}{*}{$\begin{array}{l}\text { Muslime sollten das Recht haben eigene Organisationen zu } \\
\text { gründen, um ihre Kultur zu fördern, solange sie sich an die } \\
\text { Gesetze halten. }\end{array}$} & BS & 26 & 22 & 15 & 30 \\
\hline & PH & 34 & 34 & 17 & 8 \\
\hline \multirow{2}{*}{$\begin{array}{l}\text { Frauen sollten deutlich häufiger Führungspositionen } \\
\text { übernehmen. }\end{array}$} & BS & 29 & 30 & 17 & 12 \\
\hline & $\mathrm{PH}$ & 43 & 34 & 10 & 3 \\
\hline
\end{tabular}

Autoritaristische Einstellungsmuster sind in der Gruppe der Lehrlinge weiter verbreitet als unter Lehramtsstudierenden. Bildungshintergrund und Berufswahl wirken sich hier anscheinend deutlich auf das Antwortverhalten aus. So unterschiedlich sich das Antwortverhalten auf der Ebene der deskriptiven Statistik auch darstellt, gibt es zumindest drei für die Politische Bildung relevante Gemeinsamkeiten.

Erstens: Industrie und Wissensgesellschaft stellen teilweise unvereinbare Ansprüche an die Individuen. Erstere verlangt ein hohes Maß an Disziplin und Gehorsam. Zweitere animiert zumindest teilweise zum riskanten Bruch mit Regeln und etablierten Konventionen, um Neues auszuprobieren. In beiden Studien erhöht widersprüchliches Antwortverhalten die Wahrscheinlichkeit, dass sich eine Person an der Spitze eines Staates eine Persönlichkeit wünscht, die sich nicht um Parlament und Wahlen kümmern muss. Unabhängig vom Bildungsweg scheint die Sehnsucht nach einer starken Persönlichkeit, die in der Lage ist Ordnung in die Verhältnisse zu bringen, vor allem bei jenen vorhanden $\mathrm{zu}$ sein, die sich zwischen den teilweise widersprüchlichen Anforderungen der Wissensgesellschaft hin- und hergerissen fühlen. Mehr als die Hälfte der Lehrlinge stimmen sowohl der Aussage zu, dass „Disziplin und Gehorsam in der Gesellschaft wieder wichtiger werden müssen“ als auch, dass es Leute braucht, „die Neues ausprobieren, auch wenn sie dabei Regeln verletzen“. In dieser Gruppe sehnen sich $57 \%$ nach einer starken Persönlichkeit an der Spitze eines Staates, die sich um 
demokratische Institutionen nicht weiter kümmern muss, während es in der kongruent antwortenden Gruppe nur 38 \% sind. ${ }^{37}$ Auch bei den PH-Studierenden zeigt sich, dass jene, die sich sowohl für mehr Disziplin und Gehorsam aussprechen, als auch dafür, dass Neues trotz Regelverletzungen ausprobiert werden soll, mit $48 \%$ deutlich häufiger für eine autoritäre regierende Person an der Staatsspitze sind.

Zweitens: Es besteht ein deutlicher Zusammenhang zwischen autoritaristischen Einstellungsmustern und Gruppenbezogener Menschenfeindlichkeit. Personen die einen Mangel an Disziplin und Gehorsam in der Gesellschaft diagnostizieren, härter gegen Unruhestifter vorgehen wollen, um Recht und Ordnung zu bewahren, und weniger geneigt sind, härtere Bestrafungen auch einmal auszusetzen, fühlen sich häufiger von Zuwanderung bedroht und antworten tendenziell islamfeindlicher, antisemitischer und sexistischer. ${ }^{38}$

Drittens: Lehrlinge ${ }^{39}$ und Studierende ${ }^{40}$ bevorzugen Themen der Politischen Bildung, die sich an den Lebenswelten der Lernenden orientieren, gegenüber Zeitgeschichte. Zeitgeschichtliche Themen landen unter sieben Auswahlmöglichkeiten (Aktuelle Themen, Vorurteile und Minderheitenrechte, Politische Weltanschauungen, Staat Österreich, Arbeitswelt, Parteien und Interessenvertretungen) in beiden Gruppen auf dem letzten Platz. Bei der Hälfte der Befragten landet das Thema Zeitgeschichte auf einem der beiden letzten Plätze. So wichtig das Lernen über bzw. aus Geschichte auch ist, die Arbeit gegen Antisemitismus und andere Formen Gruppenbezogener Menschenfeindlichkeit muss sich - insbesondere außerhalb des Geschichtsunterrichts zunehmend auch neue thematische Zugänge suchen, um SchülerInnen zu erreichen.

\section{Autoritarismus und Gruppenbezogene Menschenfeindlichkeit unter Jugendlichen mit muslimisch geprägtem Familienhintergrund}

Wie im vorangegangenen Abschnitt gezeigt, unterscheidet sich das Antwortverhalten zwischen Jugendlichen ab 18 an Wiener Berufsschulen und Studierenden der PH Wien in vielen Bereichen maßgeblich voneinander. Die Variablen Bildungsgrad und Berufswahl haben demnach einen relativ starken Einfluss auf Einstellungsausprägungen in den Bereichen politischer Autoritarismus, Autoritarismus, Anomie und Gruppenbezogene Menschenfeindlichkeit. Autoritäre Einstellungsmuster zeigen sich dabei in der Gruppe der BerufsschülerInnen deutlicher als unter Studierenden der PH Wien. In diesem Abschnitt gehen wir nun der Frage nach, ob ein muslimisch geprägter Familienhintergrund Einfluss auf demokratische Grundhaltungen und autoritäre

${ }^{37}$ Lauss/Schmid-Heher, Berufsschule, S. 106.

38 Ebd., S. 118; Lauss/Schmid-Heher, PH Wien, S. 12.

${ }^{39}$ Lauss/Schmid-Heher, Berufsschule, S. 114.

${ }^{40}$ Lauss/Schmid-Heher, PH Wien, S. 11. 
Einstellungen hat. Zu diesem Zweck wurden zwei Subgruppen aus der Gruppe der BerufsschülerInnen verglichen. Einerseits junge Erwachsene mit muslimischem Familienhintergrund $(\mathrm{n}=102)$, andererseits junge Erwachsene ohne Migrationshintergrund, bei denen in der Familie ausschließlich Deutsch gesprochen wird $(\mathrm{n}=310)$. Die Zuteilung erfolgte aufgrund der Antworten auf zwei Items im soziodemografischen Teil des Fragebogens. Die Vergleichsgruppe mit muslimisch geprägtem Familienhintergrund setzt sich aus Personen zusammen, in deren Haushalt ausschließlich oder zum Teil eine der folgenden Sprachen gesprochen wird ${ }^{41}$ : Arabisch, Albanisch, Bangla, Bosnisch, Dari, Farsi, Kurdisch, Somali, Tschetschenisch, Türkisch. Im Weiteren wollen wir prüfen, ob gefundene Unterschiede zwischen den Gruppen in einem Zusammenhang mit einem so definierten muslimisch geprägten Hintergrund stehen. Zu diesem Zweck haben wir eine dritte Gruppe von Personen gebildet, die angeben im eigenen Haushalt (auch) Serbisch oder Kroatisch zu sprechen ( $\mathrm{n}=88$ ).

Die Variable „muslimisch geprägter Familienhintergrund“ hat bei der großen Mehrzahl der Fragen keinen signifikanten Einfluss auf das Antwortverhalten. Sowohl die Gruppe der ausschließlich Deutschsprachigen ohne Migrationshintergrund als auch die Gruppe mit muslimisch geprägtem Familienhintergrund fühlen sich in ähnlichem Maß orientierungslos und sehen ähnlich zuversichtlich in ihre berufliche bzw. private Zukunft. Sowohl das Bekenntnis zur Demokratie als Regierungsform, als auch der Wunsch nach einer von Parlament und Wahlen unabhängigen, starken Führungspersönlichkeit ist unter BerufsschülerInnen mit und ohne Migrationshintergrund in der Familie in etwa gleich stark ausgeprägt. BerufsschülerInnen mit muslimischem Familienhintergrund bringen einer demokratisch legitimierten Regierung tendenziell mehr Vertrauen entgegen als solche ohne Migrationshintergrund. $60 \%$ (24\% sehr/36 \% eher) der Letzteren finden, dass ExpertInnen und nicht die Regierung entscheiden sollen, was das Beste für das Land ist. Nur $39 \%$ (14/25) aus der Gruppe mit muslimischem Migrationshintergrund votieren tendenziell für eine Expertokratie. Ein unterscheidbares Antwortverhalten gibt es auch bei zwei Fragen aus dem Konstrukt Autoritarismus, wobei die Unterschiede relativ gering sind: $30 \%$ (5/25) der SchülerInnen mit muslimischem Familienhintergrund finden, dass man manchmal Gnade walten lassen sollte, anstatt hart zu strafen. Befragte ohne Migrationshintergrund antworten hier in der Tendenz autoritärer. Nur 24 \% (7/17) neigen dazu, in manchen Fällen Gnade vor Recht ergehen zu lassen. Geringe Unterschiede lassen sich noch bei einer zweiten Frage aus dem Bereich Autoritarismus

\footnotetext{
41 Augenscheinlich lässt die im Haushalt gesprochene Sprache keinen direkten Rückschluss auf ein wie auch immer geartetes religiöses Bekenntnis bzw. individuelle religiöse Praxis zu. Die Gruppenkonstruktion beruht demnach auf dem Vorhandensein eines familiären Einflusses, der sich in zuhause gesprochenen Sprachen manifestiert und aufgrund der gesellschaftlichen Bedeutung von Religion als ,muslimisch geprägt' verstanden wird. Die in der Folge aufgezeigten Unterschiede sind demnach auf ein breites Spektrum von Sozialisierungsvorgängen zurückzuführen, unter denen eine solche „muslimische Prägung“ lediglich einen Faktor unter mehreren darstellt. Diese Gruppenkonstruktion erscheint insofern als aussagekräftig, als - wie in der Einleitung exemplarisch dargestellt - im gesellschaftlichen Diskurs um Antisemitismus in der Migrationsgesellschaft ZuwandererInnen aus muslimisch geprägten Herkunftsländern unabhängig von ihrem religiösen Selbstverständnis im Fokus stehen.
} 
erkennen. Hier sind allerdings SchülerInnen ohne Migrationshintergrund zu $75 \%$ (43/32) der Ansicht, dass Disziplin und Gehorsam in der Gesellschaft wieder wichtiger werden müssen. Immerhin 80 \% (46/34) der jungen Erwachsenen mit muslimischem Migrationshintergrund stimmen dieser Ansicht zumindest in der Tendenz zu.

Lediglich in einem Konstrukt finden sich durchgängig Unterschiede im Antwortverhalten zwischen Jugendlichen mit und ohne muslimischem Familienhintergrund: Gruppenbezogene Menschenfeindlichkeit. Wenig überraschend zeigen sich große Abweichungen im Antwortverhalten bei der Frage, ob Muslime das Recht haben sollten, eigene Organisationen zur Förderung ihrer Kultur zu gründen, solange sie sich an die Gesetze halten. Lediglich 38 \% der Befragten ohne Migrationshintergrund in der Familie teilen diese Einschätzung tendenziell, wohingegen in der Gruppe mit muslimisch geprägtem Hintergrund $76 \%$ der Befragten dieser Aussage zustimmen, davon nahezu alle $(66 \%)$,sehr stark“. Im Umkehrschluss bedeutet das, dass eine deutliche Mehrheit der ÖsterreicherInnen ohne Migrationshintergrund und immerhin noch ein knappes Viertel der SchülerInnen mit muslimisch geprägtem Familienhintergrund das Recht auf Versammlungsfreiheit für Muslime in Österreich einschränken wollen. In der Gruppe der Kroatisch bzw. Serbisch sprechenden Befragten befürworten 44 \% das Recht von MuslimInnen, eigenen Kulturorganisationen zu gründen.

Nicht ganz so groß, aber immer noch deutlich sind die Unterschiede bei der Frage, ob Zuwanderung im Allgemeinen gut für die österreichische Wirtschaft ist. Dies bejahen in der Tendenz fast die Hälfte (48 \%) der BerufsschülerInnen mit muslimischem Hintergrund und nicht einmal ein Drittel (26\%) der Befragten aus der Gruppe ohne Migrationshintergrund. In der dritten Gruppe von Personen, also jene mit kroatischem bzw. serbischem Hintergrund, denken $28 \%$, dass Zuwanderung überwiegend positive ökonomische Effekte hat. Auch bei einer anderen Frage, die auf Zuwanderungsängste abzielt, sind Ressentiments in der Gruppe ohne Migrationshintergrund weiter verbreitet. $52 \%$ geben an, mit Menschen aus ihrem eigenen Herkunftsland weniger Probleme zu haben als mit anderen. Mit $42 \%$ etwas weniger weit verbreitet ist dieser Eindruck unter jungen Erwachsenen mit muslimischem Familienhintergrund. Serbisch und Kroatisch sprechende Befragte antworten hier in der Tendenz ähnlich wie ihre ausschließlich Deutsch sprechenden MitschülerInnen. 


\begin{tabular}{|c|c|c|c|c|c|}
\hline $\begin{array}{l}\text { Items Gruppenbezogene Menschenfeindlichkeit: } \\
\text { ausschließlich deutschsprachige Lehrlinge ohne } \\
\text { Migrationshintergrund (a), Lehrlinge mit muslimisch } \\
\text { geprägtem Familienhintergrund } \quad \text { (b) und Lehrlinge mit } \\
\text { kroatischem bzw. serbischem Familienhintergrund } \\
\begin{array}{llll}\text { Vergleich } & \text { (c) im }\end{array}\end{array}$ & & ++ & + & - & -- \\
\hline \multirow{3}{*}{$\begin{array}{l}\text { Meine beruflichen Chancen sinken durch steigende } \\
\text { Zuwanderung. }\end{array}$} & (a) & 28 & 23 & 26 & 19 \\
\hline & (b) & 18 & 29 & 23 & 23 \\
\hline & (c) & 26 & 22 & 21 & 15 \\
\hline \multirow{3}{*}{$\begin{array}{l}\text { ZuwandererInnen sind im Allgemeinen gut für die } \\
\text { österreichische Wirtschaft. }\end{array}$} & (a) & 7 & 19 & 28 & 37 \\
\hline & (b) & 18 & 30 & 29 & 15 \\
\hline & (c) & 5 & 23 & 34 & 25 \\
\hline \multirow{3}{*}{$\begin{array}{l}\text { Mit Menschen aus meinem eigenen Herkunftsland habe ich } \\
\text { weniger Probleme als mit anderen. }\end{array}$} & (a) & 32 & 20 & 19 & 19 \\
\hline & (b) & 17 & 25 & 21 & 31 \\
\hline & (c) & 27 & 22 & 23 & 18 \\
\hline \multirow{3}{*}{ Juden haben in Österreich zu viel Einfluss. } & (a) & 10 & 6 & 23 & 43 \\
\hline & (b) & 28 & 18 & 17 & 21 \\
\hline & (c) & 24 & 16 & 17 & 25 \\
\hline \multirow{3}{*}{$\begin{array}{l}\text { Muslime sollten das Recht haben eigene Organisationen zu } \\
\text { gründen, um ihre Kultur zu fördern, solange sie sich an die } \\
\text { Gesetze halten. }\end{array}$} & (a) & 17 & 21 & 18 & 38 \\
\hline & (b) & 66 & 10 & 10 & 12 \\
\hline & (c) & 16 & 28 & 15 & 33 \\
\hline \multirow{3}{*}{$\begin{array}{l}\text { Frauen sollten deutlich häufiger Führungspositionen } \\
\text { übernehmen. }\end{array}$} & (a) & 32 & 33 & 15 & 8 \\
\hline & (b) & 23 & 25 & 20 & 19 \\
\hline & (c) & 24 & 19 & 27 & 17 \\
\hline
\end{tabular}

Während Ängste vor (weiterer) Zuwanderung unter BerufsschülerInnen mit muslimischem Hintergrund tendenziell weniger stark ausgeprägt sind, finden sexistische und antisemitische Vorbehalte anscheinend weitaus größeren Anklang. Rund zwei Drittel (65\%) der Befragten ohne Migrationshintergrund befürworten das Voranschreiten der beruflichen Gleichstellung von Männern und Frauen. In der Gruppe mit muslimischem Familienhintergrund findet sich dafür keine Mehrheit. Nur $48 \%$ stimmen der Aussage zu, dass Frauen deutlich häufiger Führungspositionen übernehmen sollten. Als besorgniserregend muss auch das Antwortverhalten bei einem Item bezeichnet werden, das auf antisemitische Vorurteile abzielt. Der Aussage, dass Juden in Österreich zu viel 
Einfluss haben, stimmen $16 \%$ der BerufsschülerInnen ohne Migrationshintergrund zu. In der Gruppe mit muslimischem Familienhintergrund vertritt nahezu die Hälfte (46\%), die Meinung, dass der Einfluss der Juden in Österreich zu groß ist. Dabei bejahten $28 \%$ die Aussage sehr stark und $17 \%$ eher. Weitere $17 \%$ haben keine Antwort abgegeben. Aber ist der muslimisch geprägte Familienhintergrund hier wirklich entscheidend? Die an Wiener Berufsschulen erhobenen Daten sprechen eher gegen diese These. Kroatisch bzw. Serbisch sprechende Jugendliche stehen der beruflichen Gleichstellung von Mann und Frau am skeptischsten gegenüber. Nur $43 \%$ finden, dass Frauen häufiger Führungspositionen übernehmen sollen. Außerdem stimmen auch in dieser Gruppe $40 \%$ dem antisemitischen Klischee zu, dass Juden in Österreich zu viel Einfluss haben. Die Daten legen nahe, dass Antisemitismus unter muslimischen Jugendlichen in Österreich ein Problem ist, dem größere Aufmerksamkeit gewidmet werden sollte. Allerdings scheint das Problem auch in Familien, in denen Kroatisch bzw. Serbisch gesprochen wird, ähnlich groß zu sein. Dieser Befund ist ein deutlicher Auftrag für die Politische Bildung in Berufsschulen. Das folgende Kapitel widmet sich der Frage, wie Gruppenbezogener Menschenfeindlichkeit im Allgemeinen und Antisemitismus im Speziellen im schulischen Kontext nachhaltig begegnet werden kann. Konventionelle, auf homogene Lerngruppen ausgerichtete und primär an der österreichischen Geschichte orientierte Vermittlungsansätze stoßen hier an ihre Grenzen.

Ohne die grundlegende Bedeutung der Auseinandersetzung mit der Geschichte des Antisemitismus und dem Holocaust als wichtige Aufgabe der Schule infrage zu stellen, liegt der Fokus in diesem Beitrag auf der Einbeziehung lebensweltlich orientierter Erfahrungen. Der im folgenden Abschnitt entwickelte Ansatz findet seinen Ausgangspunkt für das Lernen gegen Antisemitismus und andere Differenzkonstruktionen in der Schilderung und wechselseitigen Anerkennung von (durchaus sehr unterschiedlichen) Ausgrenzungserfahrungen, mit denen Jugendliche in ihrem Alltag konfrontiert sind.

\section{Diskussion der Ergebnisse und Ableitungen für die Praxis Politischer Bildung an Berufsschulen}

Das Ausmaß antisemitischer Einstellungen unter Jugendlichen mit Migrationshintergrund muss zunächst als Problem wahrgenommen werden, um pädagogische Strategien bzw. entsprechende Bildungsangebote entwickeln zu können. Für deren Aussicht auf Erfolg sind bereits die Beschreibung sowie die Kontextualisierung des Problems entscheidend. Abstand zu nehmen ist von einer Betrachtung, die geeignet ist, wiederum ausgrenzende Stereotype - im aktuellen Diskurs insbesondere gegen MuslimInnen - zu reproduzieren. Das liefe nicht nur den Prinzipien einer an demokratischen Werten orientierten Politischen Bildung entgegen, sondern wäre darüber 
hinaus aus zwei Gründen falsch. Erstens ist Antisemitismus auch in der Mehrheitsgesellschaft verwurzelt. Jeder fünfte Jugendliche ohne Migrationshintergrund (19\%) stimmt der Aussage zu, dass Juden in Österreich zu viel Einfluss haben. Wie groß der Einfluss einer gesellschaftlichen Ächtung von offenem Antisemitismus in Österreich ist, kann nicht beziffert werden und eine moralische Empörung über offenen Antisemitismus schließt latenten keineswegs aus. Zweitens ist es aus mehreren Gründen erforderlich, Antisemitismus im Zusammenhang mit anderen Formen Gruppenbezogener Menschenfeindlichkeit zu betrachten. Einerseits ist Antisemitismus gerade als „dynamisches Phänomen“42 zentral für ausgrenzende und Fremdgruppen abwertende Identitätskonstruktionen. Antisemitismus zeigt sich anschlussfähig zu verschiedenen Einstellungsmerkmalen und Weltanschauungen. Er erfüllt dabei immer die Funktion, ein auf nebulose Rechtfertigungen gestütztes Feindbild zu konstruieren und bleibt dabei als „Gerücht über die Juden“43 von Erfahrungen weitestgehend unabhängig. Andererseits würde in der Berufsschule ein ausschließlicher Fokus auf antisemitische Einstellungen von Jugendlichen mit Migrationshintergrund dem Umstand nicht gerecht, dass für Jugendliche ohne Migrationshintergrund augenscheinlich Islam- und Fremdenfeindlichkeit eine deutlich größere Rolle für ausgrenzende Identitätskonstruktionen haben.

Ein spezifisch auf MigrantInnen ausgerichtetes Bildungsangebot gegen Antisemitismus ließe sich in der Schule schon aufgrund heterogener Klassenzusammensetzungen nicht realisieren und dennoch bzw. gerade deshalb zeigt sich vor dem Hintergrund des komplexen Verhältnisses zwischen Antisemitismus und anderen Differenzkonstruktionen die Notwendigkeit, verschiedene Diskriminierungserfahrungen sowie Ausgrenzungsmuster in pädagogische Bemühungen gegen Antisemitismus einzubeziehen.

Antisemitismusprävention kann sich - insbesondere unter den spezifischen Bedingungen in der Berufsschule im Hinblick auf den fachlichen Rahmen und die zur Verfügung stehende Unterrichtszeit - nicht in einer gezielten, historisch fundierten Auseinandersetzung entfalten. Der Lehrplan für den Pflichtgegenstand Politische Bildung ist in die drei Kompetenzbereiche „Lernen und Arbeiten“, „Leben in der Gesellschaft" und „Mitgestalten in der Gesellschaft“ gegliedert. (BGBl. II Nr. 211/2016) Vielmehr muss demnach jeglichen Differenzkonstruktionen ein grundlegendes Demokratie- und Politikverständnis entgegengestellt werden, das dem Ziel der politischen Mündigkeit förderlich ist und an die Lebenswelt der Jugendlichen anknüpft. Das für die schulische Politische Bildung zentrale Kompetenz-Strukturmodell versteht politische Mündigkeit als Maßstab für die Ausformung von politischen Kompetenzen. Ebenso verlangt es, die „Berücksichtigung der Erfahrungs- und Lebenswelt der SchülerInnen [...] insofern als vorrangig anzusehen, als dadurch die Relevanz politischen

${ }^{42}$ Konitzer, Moral, S. 33.

43 Theodor W. Adorno, Minima Moralia, Frankfurt a. M. 2001, S. 200. 
Lernens für die Lernenden gesichert wird“. 44 Auf Grundlage der in Kapitel vier und fünf beschriebenen Daten wird deutlich, dass die Forderungen nach Disziplin und Gehorsam und nach Härte gegenüber UnruhestifterInnen sowohl für die Gruppe der BerufsschülerInnen als auch für die Gruppe der PH-Studierenden ein verlässlicher Prädiktor für das Ausmaß Gruppenbezogener Menschenfeindlichkeit ist. Mit Adorno ist autoritären Denkmustern einzig Mündigkeit entgegenzusetzen, „die darin besteht [...], daß die Erziehung eine Erziehung zum Widerspruch und zum Widerstand ist“ “. 45 „Lernen wegen Antisemitismus" (und ebenso wegen anderer antidemokratischer, auf Ab- und Ausgrenzung beruhender, Differenzkonstruktionen) verlangt nach einer kritischen Politischen Bildung, die sich parteilich am Demokratiepostulat orientiert, und dabei nicht nur die Möglichkeit zur Wahlbeteiligung, sondern darüber hinaus die Möglichkeit zur Teilhabe an gesellschaftlichen Auseinandersetzungen zum Ziel hat. ${ }^{46}$ Die zunehmende Bedeutung einer diffusen gesellschaftlichen Unzufriedenheit, die den vermeintlichen Wunsch nach Veränderung ebenso wie die Verteidigung althergebrachter Konventionen miteinschließt, verdeutlicht den Stellenwert von Kritik für die Demokratie. Wer nicht in der Lage ist, kritische Perspektiven gegenüber Autoritäten einzunehmen, Kritik zu formulieren und an AdressatInnen zu richten, kann empfundene Unzufriedenheit bzw. Strategien zum Umgang damit nur an Autoritäten delegieren bzw. geeignete Sündenböcke suchen.

Da auch Politische Bildung an der Berufsschule im Rahmen der Berufsausbildung im Dualen System stattfindet, stehen von Seiten der Lehrlinge Erwartungen im Hinblick auf das Arbeitsleben im Vordergrund und die Identität als Lehrling ist zentral für die Lebenswelt der BerufsschülerInnen. Der Arbeitsalltag ist von hierarchischen Verhältnissen geprägt und bietet in der Regel keine Gelegenheit zum kritischen Hinterfragen von Autoritäten. Zwar ist auch die Schule ohne Zweifel ein grundsätzlich autoritäres System, doch zu den Aufgaben der Schule zählt nicht nur die Heranbildung von jungen Menschen zu ,arbeitstüchtigen, pflichttreuen und verantwortungsbewussten Gliedern der Gesellschaft", sondern auch die Befähigung zur politischen und weltanschaulichen Aufgeschlossenheit sowie zum selbstständigen Urteil und zum sozialen Verständnis. ${ }^{47}$ Wenn Sekundärtugenden wie Fleiß und Pflichttreue die politische Mündigkeit nicht einschränken sollen, muss auch der kritischen Reflexion von Macht, Hierarchien und Diskriminierung ein Stellwert eingeräumt werden. Im Hinblick auf Diversity-Konzepte, die nicht zuletzt vor dem Hintergrund einer Migrationsgesellschaft für ein diskriminierungsfreies Miteinander stehen, unterscheidet Albert Scherr zwischen

${ }^{44}$ Reinhard Krammer, Die durch politische Bildung zu erwerbenden Kompetenzen. Ein Kompetenz-Strukturmodell, Wien 2008.

45 Adorno, Erziehung, S. 145.

${ }^{46}$ Frank Nonnenmacher, Analyse, Kritik und Engagement. Möglichkeiten und Grenzen schulischen Politikunterrichts, in: Bettina Lösch/Andreas Thimmel (Hrsg.), Kritische Politische Bildung, Ein Handbuch, Bonn 2011, S. 459-470, hier S. 465.

${ }^{47}$ Schulorganisationsgesetz, \2, Aufgabe der österreichischen Schule, BGBl. 242/1962. 
solchen Konzepten, „, die allein Unterschiede in den Blick nehmen und jenen, die Vielfalt im Zusammenhang mit Macht- und Herrschaftsverhältnissen [...] thematisieren “. ${ }^{48}$ Während in der Politischen Bildung an Berufsschulen die Aufklärung über Rechte und Pflichten von Lehrlingen zu den thematischen Säulen des Unterrichts zählt, fehlen vielfach kritische Perspektiven auf die „Fragestellung [...], wodurch Herrschaft und Autorität von der Gesellschaft als rechtmäßig anerkannt werden. “49 Der Zusammenhang zwischen Herkunft, Geschlecht und sozialökonomischem Status sowie von Stereotypen, Vorurteilen und Diskriminierung ist hier vor dem Hintergrund der lebensweltlichen Bezüge der Lehrlinge in den Mittelpunkt zu stellen. Eine auf ein kritisches Demokratieverständnis und eine Stärkung der politischen Mündigkeit ausgerichtete Politische Bildung, die an die Lebenswelt der Jugendlichen anknüpft und demokratische Teilhabe fördert, erscheint als zielführendste Strategie gegen Antisemitismus wie auch Islamfeindlichkeit, Sexismus oder andere Formen Gruppenbezogener Menschenfeindlichkeit.

Ansätzen, die dabei Antisemitismus und z. B. Islamfeindlichkeit als austauschbare Phänomene gleichsetzen oder Islamfeindlichkeit als den neuen Antisemitismus darstellen, werden dem Verhältnis von eigenen antimuslimischen Diskriminierungserfahrungen und Antisemitismus von MuslimInnen nicht gerecht. ${ }^{50}$ Trotzdem muss herausgearbeitet werden, dass Differenzkonstruktionen sowohl bei Islamfeindlichkeit als auch bei Antisemitismus eine zentrale Rolle zukommt. Als Konstrukte, die auf Fremdzuschreibungen aufbauen, ermöglichen sie die Aufwertung der Eigengruppe (,Wir") durch die Abwertung der Fremdgruppe. Vor dem Hintergrund eigener Diskriminierungserfahrungen von MuslimInnen als Minderheiten in der Migrationsgesellschaft dient der Antisemitismus der „Verschiebung des Gegners, der dann nicht die Mehrheitsgesellschaft ist, sondert etwas Drittes, etwas, das von zwei Seiten als fremd markiert wird, von der Seite der Dominanzgesellschaft wie von ihren Minderheiten“ .51 Im Hinblick auf die Vorerfahrungen und Voreinstellungen der SchülerInnen ist aus pädagogischer Perspektive von generalisierenden Vorstellungen von Antisemitismus bzw. AntisemitInnen Abstand zu nehmen. Gerade das Thema des „migrantischen Antisemitismus"52 birgt eine Gefahr. Eine solche Wahrnehmung schränkt den Handlungsspielraum ein, weil sie Stereotype reproduziert. ${ }^{53}$ Das gilt insbesondere, wenn

48 Albert Scherr, Diversity im Kontext von Machtbeziehungen und sozialen Ungleichheiten, in: GPJE (Hrsg.), Diversity Studies und politische Bildung, Schwalbach/Ts. 2008, S. 53-64, hier S. 57.

${ }^{49}$ Unterrichtsprinzip Politische Bildung, Grundsatzerlass 2015, 22.6.2015. Bundesministerium für Bildung und Frauen, [https://www.bmb.gv.at/ministerium/rs/2015_12.pdf?61edq7], eingesehen 25.10.2017.

50 Thomas Schmidinger, Antisemitismus und Islamophobie. Einige Neuerscheinungen zum Vergleich von Antisemitismus und antimuslimischen Ressentiments, in: Österreichische Zeitschrift für Geschichtswissenschaften 22 (2011), Heft 3, S. 153-165.

${ }^{51}$ Astrid Messerschmidt, Bildungsarbeit in der Auseinandersetzung mit gegenwärtigem Antisemitismus, in: Aus Politik und Zeitgeschichte 64 (2014), Heft 28-30, S. 38-44, hier S. 42.

52 Wolfram Stender, Der Antisemitismusverdacht. Zur Diskussion über einen „migrantischen Antisemitismus“ in Deutschland, in: Migration und Schule 30 (2008), Heft 3-4, S. 284-291.

53 Heike Radvan, Pädagogisches Handeln und Antisemitismus. Eine empirische Studie zur Beobachtungs- und 
PädagogInnen ohne Migrationshintergrund mit Jugendlichen mit Migrationshintergrund arbeiten. Im Unterschied zu antirassistischen Bildungsangeboten kommen MigrantInnen im Zusammenhang mit Antisemitismus nicht als Objekte der Abwertung, sondern als „Subjekte von Diskriminierung und Ausgrenzung“54 in den Blick. Die eigenen Ausgrenzungserfahrungen können allerdings in Verbindung mit einem Perspektivenwechsel zum Ansatzpunkt für Lernprozesse gegen Antisemitismus werden. In der Praxis der Politischen Bildung an Berufsschulen werden in verschiedensten Situationen und thematischen Zusammenhängen immer wieder latente oder offene antisemitische Einstellungen - von Jugendlichen mit und ohne Migrationshintergrund geäußert, die ein „Lernen gegen Antisemitismus“ im Sinne einer Intervention und Prävention notwendig machen. ${ }^{55}$ SchülerInnen müssen dabei in die Lage versetzt werden, über ihre - bloß subjektiv empfundenen oder auch intersubjektiv nachvollziehbaren - Ausgrenzungserfahrungen zu reflektieren. ${ }^{56}$ Wenn beispielsweise geäußert wird, dass „Juden“ zu viel Einfluss hätten, sind dazu zunächst Fragen aufzuwerfen: Wer ist konkret gemeint bzw. was haben "Juden“ in diesem Zusammenhang gemeinsam? Wer hat welchen Einfluss worauf und wie ist dieser Einfluss legitimiert? Worauf habe ich welchen Einfluss und welchen Einfluss hätte ich gerne bzw. sollte ich haben? Dieses Nachfragen dient dazu, die Deutungsmuster und den Orientierungsrahmen der Jugendlichen zu erschließen. Seitens der PädagogInnen ist für diese Auseinandersetzung mit den Deutungsmustern von SchülerInnen eine rekonstruktive Erkenntnishaltung gefordert, die von stereotypisierenden Zuschreibungen und moralischen Verurteilungen Abstand nimmt. Pädagoginnen müssen distanziert beobachten und dabei zugleich den Jugendlichen die in der pädagogischen Arbeit stets nötige persönliche Anerkennung, sowie ein grundlegendes Verständnisinteresse für ihre Perspektive entgegenbringen, ohne dabei antisemitischen Einstellungen bzw. Deutungen selbst Verständnis oder gar Akzeptanz entgegen zu bringen. ${ }^{57}$ So kann vor diesem Hintergrund beispielsweise ein kritischer Diskurs die Bedeutung von Demokratie als Herrschafts-, Gesellschafts- und Lebensform eröffnet werden. Als Herrschaftsform in einem Staat setzt Demokratie freie Wahlen, rechtsstaatliche Ordnung und ein Bekenntnis zu Menschrechten voraus. Demokratie als Gesellschaftsform baut auf Pluralismus, konstruktiver Konfliktregelung und einer handlungsfähigen Zivilgesellschaft. Als Lebensform verlangt Demokratie auch von jedem und jeder Einzelnen ein gewisses Maß an Toleranz, Solidarität und nicht zuletzt

Interventionsformen in der offenen Jugendarbeit, Bad Heilbrunn 2010.

${ }_{54}$ Messerschmidt, Bildungsarbeit, S. 42.

55 Szigetvari, Gerüchte, S. 9-10.

56 Schäuble, Anders, S. 427.

57 Heike Radvan, Zum Zusammenhang von Ethnisierungen und antisemitischen Konstruktionen in Beobachtungshaltungen heutiger Jugendpädagog/innen, in: Richard Gebhart/Anne Klein/Marcus Meier (Hrsg.), Antisemitismus in der Einwanderungsgesellschaft. Beiträge zur kritischen Bildungsarbeit, Weinheim-Basel, S. 144161. 
die grundsätzliche Bereitschaft zur demokratischen Teilhabe. ${ }^{58}$ Ein solcher, am Verständnis von Jugendlichen orientierter, Diskurs auf der Grundlage lebensweltlicher Erfahrungen hat das Potential, implizites Wissen zugänglich zu machen, auf Aus- und Abgrenzung beruhende Deutungsmuster zu dekonstruieren und Alternativen zu erschließen. ${ }^{59}$ Eine Belehrung durch die Vermittlung von richtigem Wissen bzw. moralisch angemessenen Perspektiven kann sich schnell als kontraproduktiv erweisen und auf eine Überwältigung hinauslaufen, die dem Schüler bzw. der Schülerin die Möglichkeit nimmt, sich ein eigenständiges Urteil zu bilden.

Bildungsarbeit ist „kein Allheilmittel gegen Antisemitismus.“60 Insbesondere subjektorientierte Bildungsangebote können niemandem aufgezwungen werden und sind deshalb auf leichte Zugänglichkeit und Attraktivität angewiesen. Die Auseinandersetzung mit Antisemitismus bei SchülerInnen mit Migrationshintergrund stellt im Hinblick auf die oben beschriebene Ausrichtung subjektorientierter Bildung hohe Anforderungen an LehrerInnen. Insbesondere dann, wenn sie häufig selbst keinen Migrationshintergrund haben. Potenzielle Probleme liegen dabei insbesondere in kulturalisierenden Zuschreibungen, die Differenzkonstruktionen zwischen der Dominanzgesellschaft als „Wir-Gruppe“ und Minderheiten reproduzieren. Bezüge zu lebensweltlichen Erfahrungen von Jugendlichen herzustellen, ist für LehrerInnen schon an sich eine Herausforderung. Im Fall von Jugendlichen mit Migrationshintergrund kommt gerade im Zusammenhang mit Antisemitismus der Einbeziehung der selbsterfahrenen rassistischen Diskriminierung eine zentrale Bedeutung zu. Lehrlinge ohne Migrationshintergrund sind zwar in den meisten Fällen nicht unmittelbar von Rassismus betroffen, aber auch ihren Perspektiven auf beispielsweise als diskriminierend empfundene Ausgrenzung von politischen Prozessen sind hier relevant.

\section{Schlussbemerkungen}

Sozialwissenschaftliche Studien haben den Anspruch dabei zu helfen, gesellschaftlich relevante Probleme genauer zu beschreiben und zu lokalisieren. Gleichzeitig besteht die Gefahr, dass statistische Aussagen wie die, dass z. B. Antisemitismus in bestimmten Bevölkerungsgruppen häufiger auftritt als in anderen, wiederum Nährboden für undifferenzierte, negativierenden Gruppenzuschreibungen werden. Umschreiben wir eine Gruppe und definieren diese als Risiko, dann verschwinden oftmals die

58 Gerhard Himmelmann, Demokratie Lernen. Als Lebens-, Gesellschafts- und Herrschaftsform, Ein Lehr- und Arbeitsbuch, Schwalbach/Ts. 2016.

59 Schäuble, Anders, S. 425

${ }^{60}$ Albert Scherr, Aufgabenstellungen, Möglichkeiten und Grenzen der Bildungsarbeit gegen Antisemitismus in der Einwanderungsgesellschaft, in: Richard Gebhart/Anne Klein/Marcus Meier (Hrsg.), Antisemitismus in der Einwanderungsgesellschaft. Beiträge zur kritischen Bildungsarbeit, Weinheim-Basel, S. 15-28, hier S. 26. 
Eigenschaften, welche die betroffenen Menschen sonst auszeichnen. Als Sicherheitsmaßnahme droht Exklusion. ${ }^{61}$

Die zentrale Frage in diesem Beitrag war, ob sich Jugendliche mit migrantisch geprägtem familiären Hintergrund in ihren Haltungen zu Demokratie und Gruppenbezogener Menschenfeindlichkeit unterscheiden. Zudem wurde der Frage nachgegangen, ob BerufsschülerInnen mit muslimisch geprägtem Familienhintergrund stärker zu bestimmten Formen Gruppenbezogener Menschenfeindlichkeit (z. B. Antisemitismus) neigen als ihre MitschülerInnen. Zu guter Letzt sollte geklärt werden, wie im schulischen Kontext mit identifizierten Gruppenunterschieden pädagogisch und didaktisch adäquat umgegangen werden kann.

Es ist festzustellen, dass autoritaristische Potentiale unter Lehrlingen stärker ausgeprägt sind als in der Vergleichsgruppe von Wiener Lehramtsstudierenden. BerufsschülerInnen aus muslimisch geprägten Familien unterscheiden sich von ihren MitschülerInnen in Bezug auf Haltungen zu Demokratie und autoritaristischen Einstellungen nicht systematisch. Bei der Ablehnung von Menschen aufgrund gruppenspezifischer Zuschreibungen unterscheidet sich jedoch das Antwortverhalten muslimischer BerufsschülerInnen von demjenigen der Gruppe ohne Migrationshintergrund. Zweitere stehen Zuwanderung insgesamt und muslimischer Zuwanderung im Speziellen eher ablehnend gegenüber. Erstere sind tendenziell reservierter gegenüber beruflicher Gleichstellung von Mann und Frau. Außerdem positionieren sie sich deutlich seltener gegen antisemitische Stereotype. BerufsschülerInnen mit muslimisch geprägtem Familienhintergrund unterscheiden sich allerdings in ihrem Antwortverhalten in diesem Bereich nicht maßgeblich von ihren ausschließlich deutschsprachigen KollegInnen ohne Migrationshintergrund. Jugendliche, die in ihrem Alltag selbst Vorurteilen ausgesetzt sind und Erfahrung mit Diskriminierung machen, sind anscheinend ihrerseits anfälliger für antisemitische Botschaften. Insbesondere dort wo eine soziale Grammatik der Härte auf Strukturen trifft, die strikte Ein- und Unterordnung verlangen und sich mit einer strikten Logik des „Wir“ und „Die“ überlappt, ist der Nährboden für unterschiedliche Arten von Rassismen, Sexismus und Antisemitismus bereitet.

Jede Form von Antisemitismus ist ein Problem für ein demokratisches Zusammenleben und kann deshalb nicht hingenommen werden. Ein betont moralischer Zugang zum Lerngegenstand Antisemitismus erscheint allerdings wenig erfolgsversprechend. Um Vorurteile - die zweifelsfrei vorhanden sind - bearbeitbar zu machen, müssen sie auch zur Sprache gebracht werden können. Auch ausschließlich historische Zugänge greifen zu kurz. Die Geschichte von Ausgrenzungen zu thematisieren, bleibt eine wichtige Aufgabe. Dies gilt natürlich insbesondere für die Geschichte des Antisemitismus und des Holocaust in Österreich und Deutschland. Nichtsdestotrotz sollten gegen Antisemitismus gerichtete Lernprozesse in einem schülerInnenorientierten Unterricht die lebensweltlichen Erfahrungen von Jugendlichen aufnehmen und zum Thema

${ }^{61}$ Ulrich Beck, Weltrisikogesellschaft, Frankfurt a. M. 2007, S. 334. 
machen. Dies gilt vor allem für die österreichische Berufsschule, wo historisches Lernen kein eigenständiger Teil des Unterrichtsfaches Politische Bildung ist.

Der hier vorgestellte Ansatz findet seinen Ausgangspunkt für das Lernen gegen Antisemitismus, Fremdenfeindlichkeit und Sexismus in der Schilderung und wechselseitigen Anerkennung von (durchaus unterschiedlichen) Ausgrenzungserfahrungen, mit denen Jugendliche in ihrem Alltag konfrontiert sind. Politische Bildung sollte dazu beitragen, reflektierte Ordnungs- und Deutungsmuster an die Stelle von ausschließenden Differenzkonstruktionen („Wir“ sind so und „die“ sind so!) treten zu lassen. Es geht um die Kritik von Verhältnissen, die durch systematisch versagte Anerkennung die menschliche Würde verletzen. ${ }^{62}$ Dies sollte geschehen, indem nahe an der Lebenswelt von jungen Menschen mit ihren jeweiligen Ausgrenzungserfahrungen gearbeitet wird. Was wir bei aller Unterschiedlichkeit gemeinsam haben, sind unsere individuellen und gesellschaftlichen Verletzungen, mit denen wir umgehen lernen müssen. Aus pädagogischer Sicht ist es wichtig, Räume zu eröffnen, in denen die Anerkennung verschiedener Diskriminierungserfahrungen möglich wird und Vorurteile abgebaut werden können.

${ }^{62} \mathrm{Vgl}$. Axel Honneth, Verdinglichung. Eine anerkennungstheoretische Studie, Frankfurt a. M. 2005, S. $46 \mathrm{ff}$. 
